\title{
Classification of uterine EMG signals using supervised classification method
}

\author{
Mohamad O. Diab, Amira El-Merhie, Nour El-Halabi, Layal Khoder
}

Hariri Canadian University, College of Engineering, Bioelectronics Engineering, Damour, Meshref, Lebanon. Email:Diabmo@,hcu.edu.lb

Received 5 July 2010; revised 19 July 2010; accepted 5 August 2010.

\begin{abstract}
Aim: The main purpose of this article is to detect any risk of preterm deliveries at an early gestation period using uterine electromyography signals. Detecting such uterine signals can yield a promising approach to determine and take actions to prevent this potential risk. Methods: The best position for the detection of different uterine signals is the median vertical axis of the abdomen. These signals differ from each other by their frequency content. Initially, simulation is done for the real detected EMG signals: preterm deliveries (PD) EMGs and deliveries at term (DT) EMGs. This is performed by applying autoregressive model (AR) of specific order to estimate AR coefficients of these real EMG signals. Finally, after calculation of the AR parameters of the two types of deliveries, we generate two types of simulated uterine contractions by using White Gaussian Noise (WGN). Frequency parameter extraction and classification are first applied on simulated signals to test the limits and performance of the used methods. The last remaining step is the classification of the contractions using supervised classification method. Results: Results show that uterine contractions may be classified using the Artificial Neural Networks (ANNs). The Simple Perceptron ANN is applied on the signals for their supervised classification into independent groups: preterm deliveries (PD) and deliveries at term (TD) according to their frequency content.
\end{abstract}

Keywords: Uterine EMG Signals; AR Model; PSD; ANN

\section{INTRODUCTION}

A most urgent challenge in healthcare currently is the phenomenon of preterm labor, or labor prior to $37 \mathrm{com}$ pleted weeks of gestation.

Although the majority of risk factors for preterm birth have been identified, the prediction models exhibit a re- latively poor performance. On the other hand, the effectiveness of tocolytic agents depends on early introduction of therapy, therefore, timely recognition of the process leading to active labor is of prime importance [1].

Preterm labor is known as the primary cause of neonatal morbidity and mortality, and it remains a major problem in obstetrical practice. The incidence of preterm delivery varies between $5 \%$ and $11 \%$ [2].

In the developed world, the rate in general has been rising slowly over the past 10 to 20 years. In New Zealand, the singleton preterm birth rate rose from $4.3 \%$ in 1980 to $5.9 \%$ in 1999 , a rise of $37 \%$. Interestingly, the rate rose by $72 \%$ in high socioeconomic groups but only by $3.5 \%$ in the most deprived groups. This is due to the effects of delayed childbearing in affluent career-women, and to the increase in assisted reproduction in that group.

Preterm labor also leaves serious impacts on economy and society as a whole. Besides, it affects the development and health of a new born as well as the health of a birth giving woman. The complications of preterm birth include significant neurological, mental, behavioural and pulmonary problems in later child's life.

Significant progress has been made in the care of premature infants, but not in reducing the prevalence of preterm birth. The cause for preterm birth is in many situations elusive and unknown. The development of effective methods to prevent or reduce the incidence of preterm birth depends largely upon finding indicators for the problem.

Uterine electromyography (EMG) has shown great promise for monitoring patients during pregnancy, so it's of great interest in parturition and pregnancy abnormality identification.

Effect of the recording electrode position on the spectral content of the signal has been investigated by using a mathematical model of the women's abdomen. Then, the simulated results have been compared to actual recordings.

On signals with noise reduced with a dedicated algo- 
rithm, the main frequency components of the signal spectrum have been characterized in order to compute parameters indicative of different situations: preterm contractions resulting nonetheless in term delivery (i.e. normal contractions) and preterm contractions leading to preterm delivery (i.e. high-risk contractions). A diagnosis system permitted to discriminate between these different categories of contractions [3].

The best position for the detection of different uterine signals is the median vertical axis of the abdomen. The frequency content of the uterine contractions changes from one woman to another and during pregnancy. The obtained signals can be classified into different groups depending on their frequency because the main analyzed parameters are extracted from the frequency content of the uterine activity bursts [4]. The difference in frequencies is a key to determination and detection of the type of delivery.

Early detection of preterm deliveries will help to reduce costs of care and will allow taking all the suitable precautions; such as, the use of tocolytic treatments to prolong the gestation i.e. fetal development in utero [5].

Another proposed method for the detection of preterm delivery is: "Uterine EMG Analysis: Time-Frequency Based Techniques for Preterm Birth Detection".

For the purpose of detection, two steps are required: the first step aims to detect all events in this signal and to identify these events by allocating them to physiological classes: contractions, fetus motions, Alvarez or Long Duration Low Frequency (LDBF) waves. The second step consists of the identification of contractions between normal contractions and preterm birth contractions.

Recently, a method based on the comparison between AR and statistical classification models is being used. The frequency content of the contraction changes from one woman to another and during pregnancy. First, an AR model is applied on the Uterine EMG signal for the calculation of the ai parameters. Then, wavelet decom- position is used to extract the parameters of each simulated contraction, and an unsupervised statistical classification method based on Fisher test is used to classify the signals. A principal component analysis projection is then used to evidence the groups resulting from this classification. Results show that uterine contractions may be classified into independent groups according to their frequency content and according to term (at the recording, or at delivery) [6].

We propose in this article a method based on AR model and ANNs to characterize the uterine contractions recorded by abdominal EMG. The Simple Perceptron ANN is applied on the signals for their supervised classification into independent groups: preterm deliveries (PD) and deliveries at term (TD) according to their frequency content (Figure 1).

\section{METHODS}

\subsection{UEMG Signals' Extraction}

The uterine EMG signals were recorded by means of two Ag-AgCl Beckman electrodes, and one reference electrode located on the patient's hip. After careful preparation of the skin (cleaning with an abrasive paste and degreasing with a mixture of ether, alcohol, and acetone), which decreases the interelectrode impedance, the electrodes are aligned directly above the median axis of the uterine muscle, on the epidermis, midway between symphysis and uterus fundus [6].

The resulting uterine EMG is amplified, band pass filtered by using a compact battery operating acquisition system.

After a thorough briefing to insure best interpatient consistency, a hand held event marker was used to record simultaneously the subject's perception of contractions and of fetal movements [6].

The information obtained by EMG recording was then analyzed on a computer with a MATLAB.

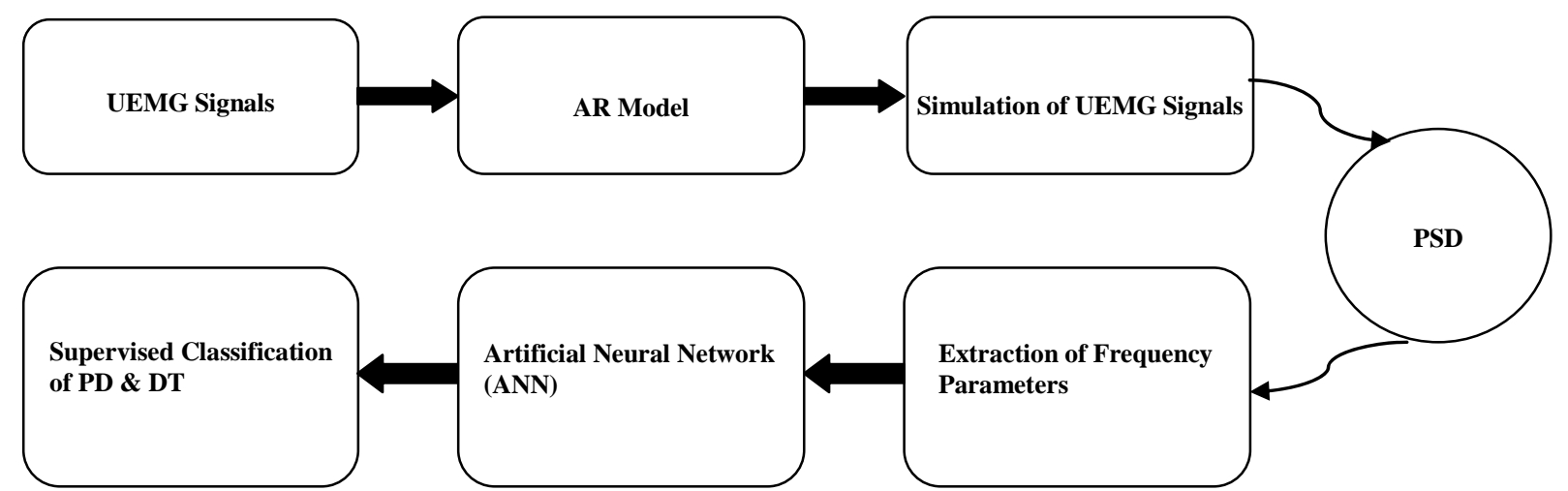

Figure 1. Block diagram showing sequence of work from obtaining uterine EMG signals, AR Modelling, simulation of original signals, extraction of frequency parameters and finally supervised classification of term and preterm labor using ANNs. 


\subsection{Modeling and Generation of Simulated UEMG Signals}

EMG(t) is the result of the integration of filtered elementary activities. Filtering induces a correlation between successive samples. An autoregressive modeling allows the detection process to be applied to the prediction error (white process) instead that to the original samples [7].

\subsubsection{Autoregressive Model (AR)}

The autoregressive model is one of a group of linear prediction formulas that attempt to predict an output $y[n]$ of a system based on the previous outputs $(y[n-1], y$ $[n-2] \ldots)$ and inputs $(x[n], x[n-1], x[n-2] \ldots)$. Deriving the linear prediction model involves determining the coefficients $a 1, a 2, .$. , and $b 0, b 1, b 2, \ldots$ in the equation:

$$
\begin{aligned}
y e[n](\text { estimated }) & =a 1 * y[n-1]+a 2 * y[n-2] \ldots \\
& +b 0 * x[n]+b 1 * x[n-1]+\ldots
\end{aligned}
$$

Several methods and algorithms exist for calculating the coefficients of the AR model, all of which are implemented by the matlab command 'ar'.

The definition being used is the following:

$$
x_{t}=\sum_{i=1}^{N} a_{i} x_{t-i}+\varepsilon_{t}
$$

Where $a_{\mathrm{i}}$ is the autoregression coefficients, $x_{\mathrm{t}}$ is the series under investigation, $\mathrm{N}$ is the order (length) of the filter which is generally very much less than the length of the series. The noise term or residue, epsilon in the above, is almost always assumed to be Gaussian white noise [8].

Several AR Models are available, in our study, we use a Linear Prediction Filter Coefficients (lpc). Lpc uses the autocorrelation method of autoregressive (AR) modeling to find the filter coefficients. It determines the coefficients by minimizing the prediction error in the least squares sense. It has applications in filter design and speech coding.

In our model and after testing many orders of AR model, we used the order 16 to estimate the AR coefficients of the two types of real EMG signals: preterm deliveries (PD) and deliveries at term (DT) (Figure 2).

After obtaining the 16 lpc coefficients for each UE-
MG signal, the mean average was calculated for these lpc coefficients of the first group (PD UEMG signals) as well as for the second group (DT UEMG signals).

After calculation of the mean average of the AR parameters (coefficients) of two groups of UEMG signals, we generated two types of simulated uterine contractions by using White Gaussian Noise (WGN) (Figure 3).

\subsection{Parameters' Extraction and Classification}

After simulation the EMG signals, power spectrum density (PSD) has to be applied on them for further extraction of frequency parameters from them.

\subsubsection{Power Spectrum Density (PSD)}

Power spectral density function shows the strength of the variations (energy) as a function of frequency. In other words, it shows at which frequencies variations are strong and at which frequencies variations are weak. Computation of PSD is done directly by the method called FFT or computing autocorrelation function and then transforming it. The goal of spectral estimation is to describe the distribution (over frequency) of the power contained in a signal, based on a finite set of data. Estimation of power spectra is useful in a variety of applications.

The power spectral density (PSD) of a stationary random process $x_{n}$ is mathematically related to the autocorrelation sequence $\left(R_{X X}\right)$ by the discrete-time Fourier transform. In terms of normalized frequency, this is given by:

$$
P_{x x}(\omega)=\frac{1}{2 \pi} \sum_{m=-\infty}^{\infty} R_{x x}(m) e^{-j \omega m}
$$

This can be written as a function of physical frequency $f$ (e.g., in hertz) by using the relation $\omega=2 \pi f \mid f_{s}$, where $f_{\mathrm{s}}$ is the sampling frequency [9].

$$
P_{x x}(f)=\frac{1}{f_{8}} \sum_{m=-\infty}^{\infty} R_{x x}(m) e^{-2 \pi j f m / f_{x}}
$$

In our study, we used periodogram. After that, frequency parameters of a signal have to be extracted. Then, we used to extract ten frequency parameters (using decile method) from each simulated signal and stored them successively in a matrix for precise and accurate classification with minimum error.

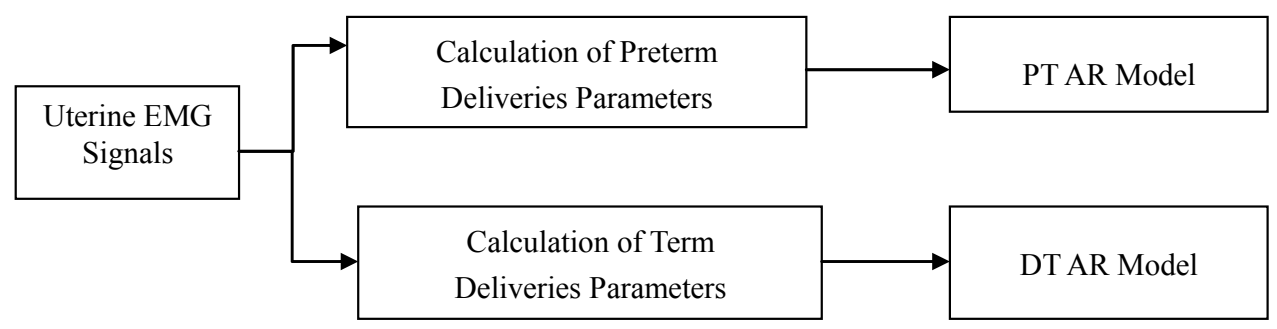

Figure 2. Creation an AR model for the PD and DT. 


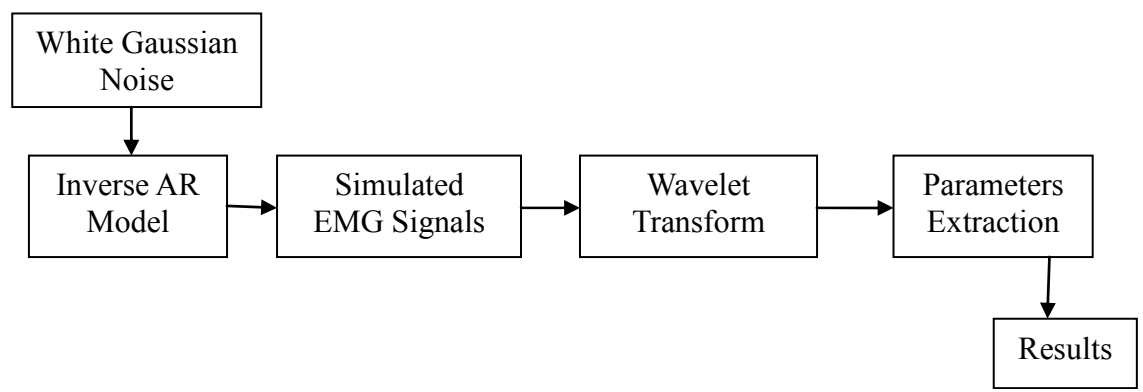

Figure 3. Block diagram for the classification of a new data.

\subsubsection{Artificial Neural Networks (ANNs)}

For the classification to be properly performed, we used artificial neural networks (ANNs).

ANNs are mathematical algorithms that are ideal for the classification of objects (e.g., patients) based upon one or more input variables (e.g., uterine EMG variables) [10]. "Artificial intelligence" is the field of computer science that attempts to give computers humanlike thought. One of the primary means by which the computers are endowed with such capability is through the use of an ANN. An ANN is composed of fundamental components, usually a circuit or computer program, which are designed to be the machine equivalent of neurons in the brains of living creatures. ANNs are made up of inputs (hidden layers) and outputs.

Most recently work has progressed which will utilize various biological and clinical markers for evaluating the risks of preterm labor using ANNs [11]. Values calculated for a number of uterine EMG parameters were used as inputs for a part for an ANN, and the outputs, specifically patient classifications, were compared to clinical assessments. The joining of uterine EMG with ANNs in this way may produce a powerful, objective tool for assessing labor.

The ANN that we used in our study is the simple perceptron neural network.

The network of the simple perceptron adapts as follows: change the weight by an amount proportional to the difference between the desired output and the actual output.

As an equation, it is represented in this way:

$$
\Delta W_{i}=\eta *(D-Y) \cdot I_{i}
$$

where $\eta$ is the learning rate, $D$ is the desired output, and $Y$ is the actual output.

Thus, we created a new simple perceptron that was trained on the frequency parameters of twenty UEMG signals representing preterm delivery and delivery at term respectively with a 100 iterations. After the successful training, the remaining extracted frequency parameters of the simulated signals ( 30 simulated signals) were intr- oduced for testing, it was found that almost all of them were classified correctly (minimum error was obtained) showing that artificial neural network is an effective classification method.

Therefore, the Simple Perceptron ANN is applied on the signals for their supervised classification into independent groups: preterm deliveries (PD) and deliveries at term (TD) according to their frequency content.

\section{FINAL RESULTS}

A successful classification of preterm labor and labor at term was achieved. We notice this classification clearly on the Figure 4:

The displayed results correspond to the simulated UEMG signals that were introduced to the simple perceptron ANN to test its validity of classification. First 15 signals correspond to group G1 which are the preterm labor contractions of the uterus while the last 15 signals are from the group $\mathrm{G} 2$ which are the uterus contractions of labor at term. It is clear from Figure 4 that the classification was achieved successfully with a minimal error.

The confusion matrix clearly demonstrates the confusion error which is equal to $3.33 \%$ which is a minimum

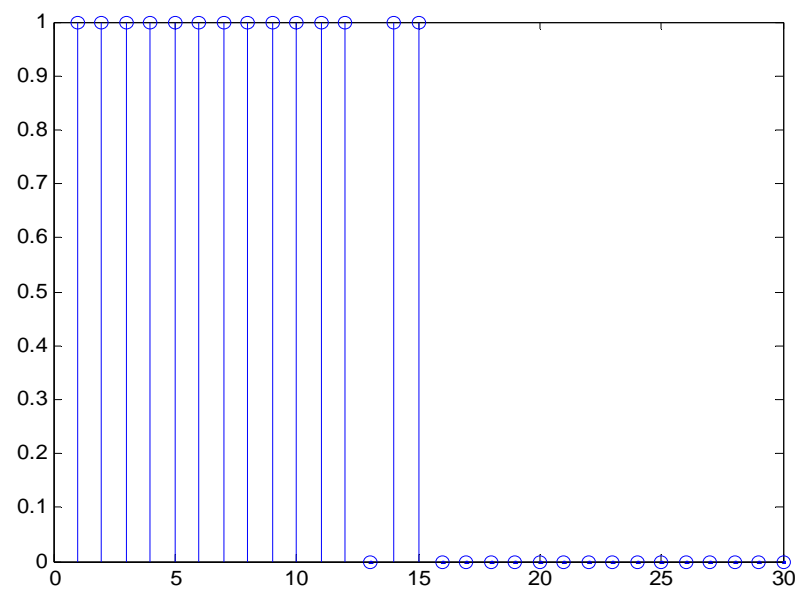

Figure 4. Results of classification of simulated UEMG signals. 
error value (Table 1). Moreover, we have run this program several times and found the mean average of the confusion errors; it was found to be equal to $1.332 \%$ for 10 runs.

After trying the extraction of 2 frequency parameters, we obtained the following Figure 5:

The confusion error is $100 \%$. Therefore, 2 frequency parameters are not enough for the classification of UEMG signals (Table 2).

\section{DISCUSSION}

In our research, we studied the uterine EMG signals of the pregnant women to be able to classify them according to the term of the delivery. The method of classification used in our study is the supervised classification method based on Artificial Neural Networks (ANNs). From the results obtained, we were able to proof that ANN (Simple Perceptron) is a good method of classification providing minimum confusion error.

Table 1. Confusion matrix: result of classification on G1 and G2.

\begin{tabular}{ccc}
\hline & G1 & G2 \\
\hline G1 & 14 & 0 \\
G2 & 1 & 15 \\
\hline
\end{tabular}

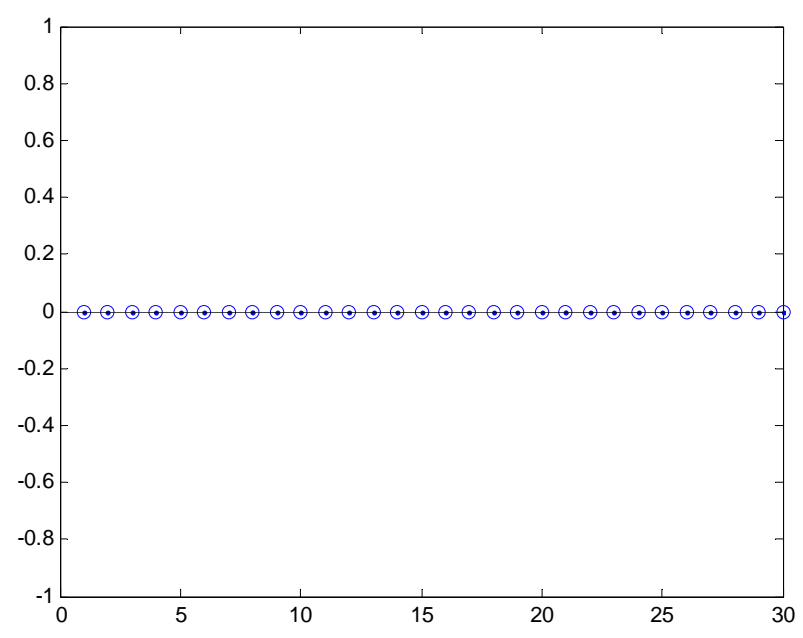

Figure 5. Results of classification of simulated UEMG signals using 2 frequency parameters.

Table 2. Confusion matrix: result of classification on G1 and G2 using 2 frequency parameters.

\begin{tabular}{ccc}
\hline & G1 & G2 \\
\hline G1 & 0 & 15 \\
G2 & 0 & 15 \\
\hline
\end{tabular}

In addition to this, we varied the number of extracted frequency parameters from each UEMG signal. We have found that as the number of parameters decreases, confusion error increases indicating that the decile extraction (10 frequency parameters) is the best of them (minimal confusion error 3.33). We have tried extracting only five and two frequency parameters from each signal; this gave less accurate results.

\section{CONCLUSIONS}

In this article, we have presented an approach for supervised classification method by using Artificial Neural Networks Simple Perceptron. Classification is based on the extraction of frequency parameters from each UEMG signal because frequency characterizes each signal. Then, signals are being classified according to differences and similarities in their frequency parameters as viewed by simple perceptron neuron.

The classification method was applied on the simulated signals, and it gave very good results. Therefore, we have found that our supervised method of classification is an efficient way to distinguish between preterm labor and labor at term.

Various clinical techniques used for this classification such as the use of fetal fibronectin and tocodynamometer (TOCO), all have a limited range of usefulness in this regard [12]. Even noticeable dynamic cervical change, long thought to be indicative, may not always be an accurate identifier of true labor, since a large percentage of women with established cervical dilation do not actually deliver preterm, even when they are not treated with laborinhibiting, or tocolytic agents [13]. Moreover, these methods do not lead predictive value above $65 \%$. Therefore, we can suggest the use of ANN as a more effective method of classification ( $96.7 \%$ predictive value).

As a perspective, we believe that an improvement might be attained by using multi layer perceptron neuron (MLP).

\section{REFERENCES}

[1] Mckean, M., Walters, A.W.W. and Smith, R. (1993) Prediction and early diagnosis of preterm labor: A critical review. Obstetrical \& Gynecological Survey, 48(4), 209-25.

[2] Senat, M.V., Tsatsaris, V., Ville, Y. And Fernandez, H. (1999) Menaced'accouchement prématuré. Encycl Méd Chir (Elsevier, Paris), Urgences, 17.

[3] Marque, C.K., Terrien, J., Rihana, S. and Germai, G. (2007) Preterm labour detection by use of a biophysical marker: The uterine electrical activity. BMC Pregnancy and Childbirth.

[4] Newman, R.B., Gill, P.J., Campion, S. and Katz, M. (1987) Antepartum ambulatory tocodynamometry: The significance of low-amplitude, high-frequency contractions. Obstetrics \& Gynecology, 70(5), 701-750. 
[5] Marque, C., Duchêne, J., Leclercq, S. And et al. (1986) Uterine EHG processing for obstetrical monitoring. IEEE Transactions on Biomedical Engineering, 33(12), 1182-1187.

[6] Diab, M.O., Marque, C. and Khalil, M.A. (2007) Classification for uterine EMG signals: Comparison between AR model and statistical classification method. International Journal of computational cognition, 5(1).

[7] Khalil, M. and Duchene, J. (1999) Detection and classification of multiple events in piecewise stationary signals: Comparison between autoregressive and multiscale approaches. Signals processing, 75(3), 239-251.

[8] Honso, H. (1996) Statistical digital signal processing and modelling. John Wiley \& Sons.

[9] Kay, S. (1988) Modern spectral estimation theory and application. englewood cliffs, Prentice-Hall, New Jersey.

[10] Gurney, K. (1997) An introduction to neural networks, University College London Press.

[11] Lockwood, C.J. and Kuczynski, E. (2001) Risk stratification and pathological mechanisms in preterm delivery. Paediatric and Perinatal Epidemiology, 15(Suppl 2), 78-89.

[12] Iams, J.D. (2003) Prediction and early detection of preterm labor. American Journal of Obstetrics \& Gynecology., 101(2), 402-412.

[13] Linhart, J., Olson, G., Goodrum, L., Rowe, T., Saade, G. and Hankins, G. (1990) Preterm labor at 32 to 34 weeks gestation: Effect of a policy of expectant management on length of gestation. American. Journal of Obstetrics \& Gynecology, 178-179. 